\title{
Probabilistic Spinning Reserve Provision Model in Multi-Control Zone Power System
}

\author{
Ali Ahmadi-Khatir, Student Member, IEEE, Mokhtar Bozorg, Student Member, IEEE, and \\ Rachid Cherkaoui, Senior Member, IEEE
}

\begin{abstract}
Inter-zonal trading in multi-area power system (MAPS) improves the market efficiency and the system reliability by sharing the resources (energy and reserve services) across zonal boundaries. Actually, each area can operate with less reserve resources than would normally be required for isolated operation. The aim of this work is to propose a model that includes the problem of optimal spinning reserve (SR) provision into the security constraint unit commitment (SCUC) formulation based on the reliability criteria for a MAPS. The loss of load probability (LOLP) and the expected load not served (ELNS) are evaluated as probabilistic metrics in the case of a multi-control zone power system. Moreover, we demonstrate how these criteria can be explicitly incorporated into the market-clearing formulation. The non-coincidental nature of spinning reserve requirement across the zonal boundary is effectively modeled. Two system cases including a small-scale (six-bus) test system and the IEEE reliability test system (IEEE-RTS) are used to demonstrate the effectiveness of the presented model.
\end{abstract}

Index Terms-Multi-control zone, probabilistic approach, reliability metrics, spinning reserve, unit commitment.

\section{NOMENCLATURE}

The main notation used throughout the paper is stated below for quick reference. Other symbols are defined as required.

\section{A. Indices and Sets}

$\begin{array}{ll}A, A A & \text { Index of control zone (Area). } \\ i j & \text { Index of generators. } \\ t & \text { Index of time periods. } \\ m n & \begin{array}{l}\text { Index of line from bus } m \text { to bus } n, \text { also used for } \\ \text { tie-lines. }\end{array} \\ \Gamma_{A} & \text { Set of tie-lines of zone A. } \\ \Omega^{A} & \text { Set of generators of zone A. } \\ \mathcal{G}_{m}^{A} & \text { Set of generators in bus } m \text { of zone A. } \\ \mathcal{B}_{m}^{A} & \text { Set of border buses connected to bus } m \text { in zone A. } \\ \Xi_{A}^{A A} & \text { Set of all tie-lines between zone A and zone } A A .\end{array}$

Manuscript received May 30, 2012; revised September 06, 2012, November 13, 2012, and December 18, 2012; accepted January 12, 2013. This work was supported by Swiss Electric Research for the project of multi-area security assessment. Paper no. TPWRS-00580-2012.

The authors are with the Power System Research Group, Ecole Polytechnique Federale de Lausanne (EPFL), Lausanne, Switzerland (e-mail: ali.khatir@epfl. $\mathrm{ch}$ ).

Digital Object Identifier 10.1109/TPWRS.2013.2243923

\section{B. Variables}

$N_{m t}^{A, c} \quad$ Net injection of bus $m$ of zone A at time $t$ in case of contingency.

$\mathbf{N}_{\mathbf{t}}^{\mathbf{A}, \mathbf{c}} \quad$ Zone A net injection vector at time $t$ in the case of contingency.

$P_{i t} \quad$ Power output of unit $i$ at time $t$.

$\mathbf{P}_{\mathrm{t}}^{\mathbf{A}} \quad$ Zone A unit power output vector at time $t$.

$R_{i t} \quad$ Spinning reserve provided by unit $i$ at time $t$.

$R_{n m, t}^{A A} \quad$ Variable for spinning reserve assistance from bus $n$ in zone $A A$ to bus $m$ in zone $A$ at time $t$.

$R_{m n, t}^{A} \quad$ Variable for spinning reserve assistance from bus $m$ in zone $A$ to bus $n$ in zone $A A$ at time $t$.

$R_{t}^{A} \quad$ Required spinning reserve for zone $A$ at time $t$; determined based on probabilistic approach.

$S_{i t}^{D} \quad$ Shut down cost of unit $i$ at time $t$.

$S_{i t}^{U} \quad$ Start up cost of unit $i$ at time $t$.

$\mathbf{T}_{t}^{A} \quad$ Zone $A$ tie-lines power flow vector at time $t$.

$\mathbf{T}_{t}^{A, c}$

Zone $A$ tie-lines power flow vector at time $t$ in the case of contingency.

$T_{m n, t}^{A} \quad$ Power flow on tie-line $m n$ of zone $A$ at time $t$; it is the negative of $T_{n m, t}^{A A}$.

$T_{m n, t}^{A, c} \quad$ Power flow on line $m n$ (including internal lines and tie-lines) of zone $A$ at time $t$ in the case of contingency; it is the negative of $T_{n m, t}^{A A, c}$.

$u_{i t} \quad$ Binary variable where 1 means unit $i$ is online at time $t$, otherwise 0 .

ELNS $_{t}^{A}$ Zone $A$ expected load not served at time $t$.

$\operatorname{LOLP}_{t}^{A}$ Zone $A$ loss of load probability at time $t$.

$\theta_{m t}^{A} \quad$ Phase angle of bus $m$ of zone $A$ at time $t$.

$\theta_{m t}^{A, c} \quad$ Phase angle of bus $m$ of zone A at time $t$ in case of contingency.

$\boldsymbol{\theta}_{t}^{A} \quad$ Zone $A$ phase angle vector at time $t$.

$\boldsymbol{\theta}_{t}^{A, c} \quad$ Zone $A$ phase angle vector at time $t$ in the case of contingency.

$\delta_{i t} \quad$ Binary variable where 1 means the outage of unit $i$ causes lost load at time $t$; otherwise 0 . 
$\delta_{i j, t} \quad$ Binary variable where 1 means the common outages of units $i$ and $j$ in the same control zone causes lost load at time $t$; otherwise 0 .

$\delta_{n m, t} \quad$ Binary variable where 1 means outage of tie-line $m n$ causes lost load in the target zone at time $t$; otherwise 0 .

$\eta_{i k, t} \quad$ Binary variable where 1 means the common outages of unit $i$ in a target zone and unit $k$ in its adjacent zone causes lost load at time $t$; otherwise 0 .

\section{Parameters and Constants}

B $^{\mathbf{A}} \quad$ Zone $A$ network admittance matrix; zone A tie-lines are ignored.

$\mathbf{D}_{\mathbf{t}}^{\mathbf{A}} \quad$ Zone $A$ bus load demand vector at time $t$.

$D_{m t}^{A} \quad$ Load demand of bus $m$ of zone $A$ at time $t$.

$D_{t}^{A} \quad$ Zone $A$ total load demand; it is the summation of all buses' demand in zone $A$ at time $t$.

$\mathbf{H}^{\mathbf{A}} \quad$ Zone $A$ node to tie-line incidence matrix.

$L_{m n}^{A, \max } \quad$ Maximum transfer capability of internal transmission line $m n$ of zone $A$.

$P_{i}^{\max } \quad$ Maximum capacity of unit $i$.

$P_{i}^{\min } \quad$ Minimum capacity of unit $i$.

$q_{i t} \quad$ Price offer of unit $i$ for spinning reserve at time $t$.

$R_{i}^{\max } \quad$ Ramp-up rate limit of unit $i$.

$T_{m n}^{A, \text { rmax }} \quad$ Maximum transfer capability of tie-line $m n$.

$\overline{T_{m n}^{A, c}}$

$x_{m n}$

$\overline{\mathrm{ELNS}}_{t}^{A}$

$\overline{\mathrm{LOLP}}_{t}^{A}$

Transmission line $m n$ emergency rating limit.

Reactance of line $m n$.

Predefined ceiling value for ELNS of Zone $A$ at time $t$.

Predefined ceiling value for LOLP of Zone $A$ at time $t$.

$\mathcal{P}_{i t}^{1} \quad$ Outage probability of unit $i$ in zone $A$ at time $t$.

$\mathcal{P}_{i j, t}^{2} \quad$ Double outage probability of units $i$ and $j$ in target zone $A$ at time $t$.

$\mathcal{P}_{m n, t}^{A, A A} \quad$ Outage probability of tie-line $m n$ between zone $A$ and $A A$ at time $t$.

$\mathcal{P}_{i k, t}^{A, A A} \quad$ Common outage probability of unit $i$ in zone $A$ and unit $k$ in zone $A A$ at time $t$.

\section{INTRODUCTION}

\section{A. Motivation and Aim}

$\mathbf{S}$ PINNING reserve is the unloaded section of the synchronized power which is able to respond immediately in case of contingencies. It is a critical resource maintained by the power system operator in order to keep up the security in case of unforeseen events such as, generation outages and sudden load changes. The system security can be improved by increasing the system generating reserve. This, however, increases the system operating cost [1].

Now, the principal question raised here is what is the optimal amount of spinning reserve that should be provided? The best answer is that the required reserve in the system should be provided in such a way that the reserve costs and benefits in terms of system reliability are balanced.

Deterministic and probabilistic techniques can be both used to establish spinning reserve requirements. The deterministic approaches set spinning reserve to a predefined amount equal to the capacity of the largest online unit, to some fraction of the peak load or to any combination of both. Although these techniques can be understood and implemented easily, they do not explicitly and accurately reflect the actual system risk with which the operator is faced. As a result, they could lead to over or under scheduling which respectively could be uneconomic or unreliable. Probabilistic techniques, however, can provide a comprehensive and realistic evaluation of the risk by incorporating the stochastic nature of the system components and the load behavior [2] and [3].

Inter-zonal trading in MAPS improves the system reliability by sharing the resources (energy and reserve services) across zonal boundaries; it positively affect the market efficiency as well [2] and [4].

The aim of this work is to propose a market-clearing model that includes the problem of optimal spinning reserve provision into the SCUC formulation based on the reliability criteria for a MAPS.

Two distinct approaches to analyze MAPS can be considered, where the first one is the centralized and the second one is the decentralized approach [2]. In the first approach, a central operator with a full view of the network operates the large MAPS. Every regional operator sends the required data regarding its own network to the central operator. On the other hand, in the decentralized approach, there is no central operator and the system operation is shared among regional operators that are each responsible for their own respective area.

The focus of the present paper will be on the centralized MAPS, which for the sake of brevity, will be referred to as "multi-control zone" throughout the paper.

\section{B. Literature Review and Contribution}

Due to inter-area tie-lines constraints, solving the SCUC problem (to schedule generation and spinning reserve) in a MAPS is much more complicated than in the case of a single-area power system. Nevertheless, it is useful to carry out a literature survey that explores the existing methods in a single-area power system.

The idea behind using probabilistic criteria for spinning reserve provision is not entirely new [5]. Two methodologies designated respectively statistic-based (performing statistical analysis and without solving the optimization problem) and optimization-based (along with solving the optimization problem) can be employed in the probabilistic reserve provision. The application of statistic-based reserve provision requires statistical assessment of all drivers making imbalance between generation 
and consumption so that a target risk level is ultimately satisfied. These drivers include power plant outage, load demand variation, load forecast errors, forecast errors of intermittent generation, and the change in exchange schedules (e.g., [4], [6], and [7]).

Contrary to the statistic-based reserve provision method, the optimization-based methodology incorporates the risk index into an optimization problem, and then the optimum amount of required reserve is achieved by solving this problem. One of the first studies in which a optimization-based probabilistic technique was used for an implicit spinning reserve assessment is due to Guy [8]. He used the PJM method proposed in [5] for economic scheduling of generating units, based on the principle that it satisfies a predefined risk target. Later, several authors, e.g., [9]-[11] and [12], used an iterative approach to incorporate the risk criteria into the reserve provision problem. The problem was solved in two stages; at the first stage, the optimization problem is solved with initial reserve; in the second stage, the risk index is calculated with the results coming from the optimization problem (post-processing). If the realized risk is not within a certain range of a pre-specified target, the initial reserve are updated, and the optimization problem is run again. Obviously, this could be computationally expensive, since it may require that the optimization problem be solved several times. Searching technique addressed in [13] can be used to expedite the updating process and subsequently decrease the computation time.

The aforementioned references suffer from sub-optimality, since they do not represent efficiently the probability distribution of the discrete capacity outage directly in terms of UC variables. To cope with this problem, [14] proposed an approximate function for LOLP index, whose parameters are system-dependent, to integrate this reliability metric into the UC problem as a continuous inequality constraint. Reference [15] penalized the ELNS cost into the objective function of the UC problem. ELNS metric is approximated using a function resulting from commitment of a particular combination of generating units. The approximated ELNS must be calibrated for each load level. Although post-processing of the results and iterations are avoided, the obtained results of [14] and [15] are not always enough accurate.

To circumvent the above difficulties, [16] and [17] include the risk index into the $\mathrm{UC}$ formulation as a continuous variable such that its mathematical form is compatible with the mixed integer linear programming (MILP) algorithm. This methodology was only applied for a single period of network-free single area power system. We follow the idea of [16] in integrating the reliability metrics into the proposed market-clearing formulation to deal with the essential issues of multi-control zone power system.

Note that all the aforementioned literature deal with approaches determining the required reserve implicitly. On the other hand, [18]-[20] and [21] employ a two-stage stochastic programming technique to provide the required reserve explicitly. Unlike the implicit reserve provision approaches, in the explicit reserve provision model, the reserve requirements are taken into account by possible realization of different scenarios and also the physical locations of contingency in the network are effectively considered. However, the difficulty with stochastic programming is that the problem size and the computational time increases with the number of scenarios since a large number of scenarios are often required to ensure the quality of the solution. Ruiz et al. combined both implicit and explicit approaches for reserve management in [22]. The rational of this study was the few scenarios in explicit approach cannot capture the whole spectrum of uncertainty. They stated that the enforcement of implicit method on the scenarios serves a further confidence for the risk averse operators.

Needless to say that both models (implicit and explicit reserve provision) are two different well-known approaches that are used in the literature for the reserve assessment.

It is worth noting that although applying probabilistic techniques for spinning reserve provision has been well developed for single area power systems, this subject is weakly investigated in MAPS. Nonetheless, several papers based on deterministic approach have been reported in the literature [23]-[25] and [26] to solve spinning reserve scheduling problem in a MAPS.

Within the above context, the contribution of this paper is threefold:

1) To determine implicitly the optimal level of spinning reserve in a multi-control zone power system; two established metrics of LOLP/ELNS approximation and SCUC formulation in an unified framework are considered.

2) To extend the reliability metrics calculation for a multicontrol zone power system while the power flow injections in the area borders are accounted for.

3) To model the non-coincidental nature of spinning reserve requirement by introducing the notation of artificial contingency load flow associated with each control zone.

\section{Paper Organization}

The rest of the paper is organized as follows. Section II presents the mathematical formulation of market-clearing problem, i.e., SCUC formulation, (Section II-A), describes the methodology for reliability metrics calculation (Section II-B), and describes how the non-coincidental nature of spinning reserve requirement is modeled (Section II-C). Section III presents and discusses the results for a six-bus test system and the IEEE-RTS. Conclusions drawn from the study are provided in Section IV. Finally, the linearization carried out is explained in the Appendix.

\section{Proposed Model Formulation}

\section{A. Market-Clearing Problem}

The centralized market-clearing problem for the entire interconnected power system, including power and spinning reserve scheduling, is formulated as a mathematical optimization problem as follows:

$$
\min \sum_{A} \sum_{i \in \Omega^{A}} \sum_{t \in T}\left(C_{i}\left(P_{i t}, u_{i t}\right)+S_{i t}^{U}+S_{i t}^{D}+q_{i t} R_{i t}\right),
$$

The above objective function is composed of the generators production costs, the startup and shutdown costs and the costs for spinning reserve provided in a multi-control zone power 
system. The generators'production cost, $C_{i}\left(P_{i t}, u_{i t}\right)$, is considered a conventionally quadratic function. For expressing in linear form, this cost function can be approximated by a set of piecewise segments as addressed in [27]. The startup and shutdown costs are considered to be exponential functions of off-time and on-time, respectively, of a generating unit [1].

The objective function (1) needs to be minimized over the scheduling horizon subject to the following constraints:

$$
\mathbf{B}^{\mathbf{A}} \boldsymbol{\theta}_{\mathbf{t}}^{\mathbf{A}}+\mathbf{H}^{\mathbf{A}} \mathbf{T}_{\mathbf{t}}^{\mathbf{A}}=\mathbf{P}_{\mathbf{t}}^{A}-\mathbf{D}_{\mathbf{t}}^{\mathbf{A}}, \text { for all time } t
$$

and for all zones

$\theta_{\mathrm{ref}, t}^{A}=0$, only for the zone including

slack bus (reference zone)

$\sum_{i \in \Omega^{A}} u_{i t} P_{i}^{\max } \geq D_{t}^{A}+R_{t}^{A}+\sum_{m n \in \Gamma_{A}} T_{m n, t}^{A}$, for all

time $t$ and for all zones

$\sum_{i \in \Omega^{A}} u_{i t} P_{i}^{\mathrm{min}} \leq D_{t}^{A}+\sum_{m n \in \Gamma_{A}} T_{m n, t}^{A}$ for all time $t$

and for all zones

$u_{i t} P_{i}^{\min } \leq P_{i t} \leq u_{i t} P_{i}^{\max }$, for all time $t$,

for all units of zone $A$ and for all zones

$0 \leq R_{i t} \leq u_{i t} P_{i}^{\text {max }}-P_{i t}$, for all time $t$, for all units

of zone A and for all zones

$0 \leq R_{i t} \leq R_{i}^{\max }$, for all time $t$, for all units

of zone $\mathrm{A}$ and for all zones

$\left|\frac{1}{x_{m n}}\left(\theta_{m t}^{A}-\theta_{n t}^{A}\right)\right| \leq L_{m n}^{A, \max }$, for all time $t$, for all

internal lines $m n$ of zone $\mathrm{A}$ and for all zones

$\left|T_{m n, t}^{A}\right| \leq T_{m n}^{A, \max }$, for all time $t$, for all

tie - lines $m n$ of zone A, and for all zones

$\frac{1}{x_{m n}}\left(\theta_{m t}^{A}-\theta_{n t}^{A A}\right)-T_{m n, t}^{A}=0$, for all time $t$

for all tie - lines $m n$ of zone A, and for all zones

$\sum_{i \in \Omega^{A}} R_{i t}+\sum_{m n \in \Gamma_{A}} R_{n m, t}^{A A}=R_{t}^{A}$, for all time $t$,

considering all tie - lines $m n$ of zone A and for all zones

$\sum_{m n \in \Gamma_{A}} R_{m n, t}^{A} \leq \sum_{i \in \Omega^{A}} R_{i t}$, for all time $t$ and for all zones

$h_{i}\left(P_{i t}, u_{i t}\right) \leq 0$, for all time $t$ and for all units

of zone $\mathrm{A}$ and for all zones

$\operatorname{LOLP}_{t}^{A} \leq \overline{\operatorname{LOLP}}_{t}^{A}$, for all time $t$, for all zones

$\operatorname{ELNS}_{t}^{A} \leq \overline{\operatorname{ELNS}}_{t}^{A}$, for all time $t$, for all zones.

Here, the ultimate decision variables for each zone are $u_{i t}$, $P_{i t}$ and $R_{i t}$. Constraint (2) represents the DC nodal power balance equation for zone A; this constraint indicates that the load demands of zone A are to be supplied by both local generation within the area and the energy that may be imported from the neighboring control zones. (3) defines the system slack bus for the reference zone. Constraints (4) and (5) enforce that adequate units in zone $\mathrm{A}$ are committed in order to meet its required load and spinning reserve while the tie-line flows belonging to this area are respected. Equation (6) accounts for the maximum and minimum capacity limit of each unit, while (7) restricts the amount of spinning reserve at time $t$ to be less than or equal to the difference between the maximum capacity $P_{i}^{\max }$ and the scheduled power output $P_{i t}$. Constraint (8) models the possible ramping restrictions of spinning reserve. Equation (9) represents the internal transmission line power flow limits. Equation (10) represents zone A tie-line power flow limits, and (11) are zone A tie-line power flow equations. Note that, (12) specifies the spinning reserve balance constraint in a given zone A; in fact, the spinning reserve requirement for each zone is met through a combination of local and imported zonal spinning reserve from adjacent areas. Note that, unlike power demand requirement which is concurrent across all control zones, the spinning reserve requirements are typically non-coincidental, and the interpretation of spinning reserve balance constraint and inter-zonal reserve variable, $R_{n m . t}^{A A}$, need more consideration. To deal with this issue, additional constraints derived from the artificial contingency load flow, described in Section II-C, are taken into account. (13) implies that the amount of spinning reserve that zone A can provide to the other zones is constrained by the maximum available spinning reserve in this zone. Equation (14) is describing the technical constraints of generators such as startup and shutdown costs linear approximation, minimum up/down time restriction, ramping up/down time limits, etc. For the detailed presentation of all the mathematical expressions of these constraints, the reader is referred to [27]. Constraints (15) and (16) are the reliability boundary constraints. Actually, by imposing the reliability metrics in a zone to be less than the predefined boundary values, the spinning reserve requirement can be set implicitly. The boundary values are specified based on the desired degree of system risk and the conditions under which the system is being operated by a regulatory agency; they are considered to be known in the proposed model.

References [13] and [18] discussed about advantages and disadvantages of ELNS and LOLP, and pointed out effectively that reliability metrics can be incorporated in a market-clearing procedure in three different fashions. The first one is to impose bounds on reliability metrics. The second way is to add a penalty function, increasing monotonically with ELNS, to the objective function of the market-clearing problem through the so-called value of lost load (VOLL). The third criterion is just a combination of the two previous ones. Note that for simplicity in the market-clearing formulation presented in this paper, we just incorporate reliability metrics into the model based on imposing bound on ELNS index. It should also be noted that the proposed model is flexible such that these three fashions can be easily settled on.

\section{B. Reliability Metrics Calculation}

LOLP and ELNS are two acknowledged metrics for the evaluation of the system risk. If the unit schedules (unit statuses and outputs) are known, they can be easily calculated based on capacity outage probability table (COPT) [4]. If the unit schedules cannot be determined a priori, these metrics can be explicitly formulated by unit commitment variables based on every possible outage events. These classical reliability measure of LOLP and ELNS for a given control zone A can be evaluated as 


$$
\begin{aligned}
\mathrm{LOLP}_{t}^{A} & \sum_{i \in \Omega^{A}} \delta_{i t} \cdot u_{i t} \cdot \mathcal{P}_{i t}^{1}+\sum_{i \in \Omega^{A}} \sum_{j>i \in \Omega^{A}} \delta_{i j, t} \cdot u_{i t} \cdot u_{j t} \cdot \mathcal{P}_{i j, t}^{2} \\
& +\sum_{m n \in \Gamma_{A}} \delta_{m n, t} \cdot \mathcal{P}_{m n, t}^{A, A A} \\
& +\sum_{i \in \Omega^{A}} \sum_{A A} \sum_{k \in \Omega^{A} A} \eta_{i k, t} \cdot u_{i t} \cdot u_{k t} \cdot \mathcal{P}_{i k, t}^{A, A A}+\ldots \\
\mathrm{ELNS}_{t}^{A} & \sum_{i \in \Omega^{A}} \delta_{i t} \cdot u_{i t} \cdot \mathcal{P}_{i t}^{1}\left(P_{i t}+R_{i t}-R_{t}^{A}\right) \\
& +\sum_{i \in \Omega^{A}} \sum_{j>i \in \Omega^{A}} \delta_{i j, t} \cdot u_{i t} \cdot u_{j t} \cdot \mathcal{P}_{i j, t}^{2} \\
& \times\left(P_{i t}+R_{i t}+P_{j t}+R_{j t}-R_{t}^{A}\right) \\
& +\sum_{m n \in \Gamma_{A}} \delta_{m n, t} \cdot \mathcal{P}_{m n, t}^{A, A A}\left(T_{n m, t}^{A A}+R_{n m, t}^{A A}-R_{t}^{A}\right) \\
& +\sum_{i \in \Omega^{A}} \sum_{A A} \sum_{k \in \Omega^{A A}} \eta_{i k, t} \cdot u_{i t} \cdot u_{k t} \cdot \mathcal{P}_{i k, t}^{A, A A} \\
& \times\left(P_{i t}+R_{i t}+\sum_{n m \in \Xi_{A}^{A A}} R_{n m, t}^{A A}-R_{t}^{A}\right)+\ldots
\end{aligned}
$$

where $\mathcal{P}_{i t}^{1}, \mathcal{P}_{i j, t}^{2}, \mathcal{P}_{m n, t}^{A, A A}$ and $\mathcal{P}_{i k, t}^{A, A A}$ are, respectively, the outage probability of unit $i$ in zone $A$ at time $t$, the common outage probability of units $i$ and $j$ in zone $A$ at time $t$, the outage probability of tie-line $m n$ between zone $A$ and $A A$ at time $t$, and the common outage probability of unit $i$ in zone $A$ and unit $k$ in zone $A A$ at time $t$. The detail computation of these parameters can be found in [4].

$\delta_{i t}$ is a set of binary variables denoting whether the outage of unit $i$ at time $t$ causes some loss of load or not in zone $A$. If the unavailability of this unit leads to some load deficiencies, this variable takes the value of 1 , otherwise it takes 0 ; i.e., $\left(R_{t}^{A}<\right.$ $\left.P_{i t}+R_{i t} \leftrightarrow \delta_{i t}=1\right)$. It is shown in [28] that this relation can be written by logical constraints as shown in (19)-(20):

$$
\begin{aligned}
\delta_{i t} \cdot m_{i} & \leq\left(R_{t}^{A}-P_{i t}-R_{i t}\right) \\
M_{i}\left(1-\delta_{i t}\right) & \geq\left(R_{t}^{A}-P_{i t}-R_{i t}\right)+\epsilon
\end{aligned}
$$

where $M_{i}$ and $m_{i}$ are the upper and lower bounds, respectively, for the constraint $R_{t}^{A}-P_{i t}-R_{i t}<0$. Such bounds can usually be derived from the knowledge of the problem. It is important for the bounds to prevent unwarranted restrictions. Computationally, it is desirable for them to be as tight as possible. In fact a value of $\mathrm{M}$ as small as possible ( $\mathrm{m}$ as large as possible) is of central importance, as this affects the amount of computation in the integer programming (IP) algorithm [29].

Here, for the constraint $R_{t}^{A}-P_{i t}-R_{i t}<0, m_{i}$ may be taken as $-P_{i}^{\max }\left[\right.$ lower bound on $\left.-\left(P_{i t}+R_{i t}\right)\right]$ as $R_{t}^{A}$ goes to zero; $M_{i}$ may be taken as $\left(\sum_{\substack{j \in \Omega A \\ j \neq i}} P_{j}^{\max }+\sum_{m n \in \Gamma_{A}} T_{m n}^{A, \max }\right)$ (upper bound on variable $\left.R_{t}^{A}\right)$ as $\left(P_{i t}+R_{i t}\right)$ goes to zero.

Note that we do not normally use the strict inequalities constraints in LP and IP problem. However, for strict inequalities, we may only be able to give the least upper bound or the greatest lower bound. We can approximate $R_{t}^{A}-P_{i t}-R_{i t}<0$ using " $\leq$ " and with a suitable small number $\epsilon$ as $R_{t}^{A}-P_{i t}-R_{i t}+\epsilon \leq$ 0 . Therefore, the above description gives the following constraints representation of (19)-(20):

$$
\begin{aligned}
& -\delta_{i t} \cdot P_{i}^{\max } \leq\left(R_{t}^{A}-P_{i t}-R_{i t}\right) \\
& \left(\sum_{\substack{j \in \Omega^{A} \\
j \neq i}} P_{j}^{\max }+\sum_{m n \in \Gamma_{A}} T_{m n}^{A, \max }\right)\left(1-\delta_{i t}\right) \\
& \quad \geq\left(R_{t}^{A}-P_{i t}-R_{i t}\right)+\epsilon .
\end{aligned}
$$

Similarly, binary variable $\delta_{i j . t}$ is used in the following formulation for double generator outages for $j>i, j, i \in \Omega^{A}$. This variable is characterized by (23)-(24):

$$
\begin{aligned}
& -\delta_{i j, t}\left(P_{i}^{\max }+P_{j}^{\max }\right) \\
& \quad \leq\left(R_{t}^{A}-P_{i t}-R_{i t}-P_{j t}-R_{j t}\right) \\
& \left(\sum_{\substack{k \in \Omega^{A} \\
k \neq i, j}} P_{k}^{\max }+\sum_{m n \in \Gamma_{A}} T_{m n}^{A, \max }\right)\left(1-\delta_{i j, t}\right) \\
& \quad \geq\left(R_{t}^{A}-P_{i t}-R_{i t}-P_{j t}-R_{j t}\right)+\epsilon .
\end{aligned}
$$

Binary variables $\delta_{n m, t}$ model the outage of tie-line $m n$ at time $t$. Note that by the outage of tie-line $m n$, not surprisingly, we will lose the power $T_{n m, t}^{A A}$ and the reserve $R_{n m, t}^{A A}$ provided from zone $A A$ to zone $A$ through tie-line $m n$. This variable is characterized by (25)-(26):

$$
\begin{aligned}
& -\delta_{m n, t} T_{m n}^{A, \max } \leq\left(R_{t}^{A}-R_{n m, t}^{A A}-T_{n m, t}^{A A}\right) \\
& \left(\sum_{i \in \Omega^{A}} P_{i}^{\max }+\sum_{\substack{k l \in \Gamma_{A} \\
k l \neq m n}} T_{k l}^{A, \max }\right)\left(1-\delta_{m n, t}\right) \\
& \geq\left(R_{t}^{A}-R_{n m, t}^{A A}-T_{n m, t}^{A A}\right)+\epsilon .
\end{aligned}
$$

Also, binary variable $\eta_{i k, t}$ represents the presence or absence of some loss-of-load in target zone $A$ due to simultaneously common generator outages in zone $\mathrm{A}$ and its adjacent zone $A A$ for $i \in \Omega^{A}$ and $k \in \Omega^{A A}$. This variable can be addressed by (27)-(28) under a mild assumption, which is expected to hold in reality; the areas do not assist their neighbors by suppling the spinning reserve in the case they also experience an outage in their own regions:

$$
\begin{aligned}
& -\eta_{i k, t}\left(P_{i}^{\max }+\sum_{n m \in \Xi_{A}^{A A}} T_{m n}^{A, \max }\right) \\
& \leq\left(R_{t}^{A}-P_{i t}-R_{i t}-\sum_{\substack{n m \in \Xi_{A}^{A A} \\
n m, t}} R_{n A}^{A A}\right) \\
& \left(\sum_{\substack{j \in \Omega A \\
j \neq i}} P_{j}^{\max }+\sum_{\substack{m n \in \Gamma_{A}^{A} \\
m n \notin \Xi_{A}^{A A}}} T_{m n}^{A, \max }\right)\left(1-\eta_{i k, t}\right)
\end{aligned}
$$




$$
\geq\left(R_{t}^{A}-P_{i t}-R_{i t}-\sum_{n m \in \Xi_{A}^{A A}} R_{n m, t}^{A A}\right)+\epsilon
$$

The above formulations suffer from the nonlinear behavior of the reliability metrics as they are the products of continuous and binary variables. In the Appendix, it is illustrated that the nonlinear metrics can be re-expressed into mathematically equivalent linear equalities and inequalities of the decision variables.

It is also recognized from (17) and (18) that these two metrics are not computationally suitable, because multiple-outage events have to be taken into account to represent the reliability metrics more accurately. It is worth noting that multiple-outage events with more than two outages can be straightforwardly accommodated in reliability metrics formulation; however, to decrease the computational burden, only single and double outages are considered, and the third and higher order outages are neglected due to their low probabilities.

\section{Artificial Contingency Load Flow Constraints}

Power demand requirements are coincidental over all control zones; they are, hence, additive in principle (i.e., they are added up to form the total power demand). Instead, spinning reserve requirements are typically non-coincidental and non-additive; in other words, spinning reserve flows across the tie-lines once it is required (e.g., in the case that a contingency occurs in a given control zone and there is not enough spinning reserve in that zone to respond to this event). Generally speaking, this issue is referred to non-coincidental nature of spinning reserve requirement.

Accordingly, to represent the non-coincidental nature of spinning reserve requirement, we propose an artificial contingency load flow related to each control zone where it is presumed that an artificial contingency equal to the total required spinning reserve $\left(R_{t}^{A}\right)$ occurs in this zone. Artificial contingency load flow related to zone A defines how the reserve requirement of zone $\mathrm{A}$ is satisfied while the imported spinning reserve flows from the other zones (i.e., adjacent areas of zone A) across the borders are feasible; the reserve requirements of all other zones are to be treated as non-coincidental to zone A.

To mathematically model the contingency load flow constraints, the net injection of the border buses of the contingency zone (here, zone A) and its neighbors (all zones AA) are modified. The imported spinning reserve from the border buses of neighboring zones (bus $n$ ) to the border bus of the contingency zone (bus $m$ ), $R_{n m, t}^{A A}$, is added into the load of bus $m$ and concurrently this value is added into the resource of bus $n$. Regarding the load balance (2), the contingency load flow relations for zone A are expressed as follows:

$N_{m t}^{A, c}=\sum_{i \in \mathcal{G}_{m}^{A}} P_{i t}-D_{m t}^{A}-\sum_{n \in \mathcal{B}_{m}^{A}} R_{n m, t}^{A A}$, for all

time $t$ and all border bus $m$ of target zone A

$N_{n t}^{A A, c}=\sum_{i \in \mathcal{G}_{n}^{A A}} P_{i t}+R_{n m, t}^{A A}-D_{n t}^{A A}$, for all time $t$

and for all border bus $n$ of adjacent areas, $A A$, connected to bus $m$ in target zone A via tie - line $m n$; i.e, $\forall n \in \mathcal{B}_{m}^{A}$
$N_{l t}^{A, c}=\sum_{i \in \mathcal{G}_{l}^{A}} P_{i t}-D_{l t}^{A}$, for all time $t$, and for all

internal bus 1 in zone $A$, and for all zones $\mathbf{B}^{\mathbf{A}} \boldsymbol{\theta}_{\mathbf{t}}^{\mathbf{A}, \mathbf{c}}+\mathbf{H}^{\mathbf{A}} \mathbf{T}_{\mathbf{t}}^{\mathbf{A}, \mathbf{c}}=\mathbf{N}_{\mathbf{t}}^{\mathbf{A}, \mathbf{c}}$ for all time $t$, for all zones

$\theta_{r e f, t}^{A, c}=0$, only for reference zone

$\frac{1}{x_{m n}}\left(\theta_{m t}^{A, c}-\theta_{n t}^{A A, c}\right)-T_{m n, t}^{A, c}=0$, for all time $t$,

for all lines $m n$ (including internal lines and tie - lines)

of zone $\mathrm{A}$, and for all zones

$\left|T_{m n, t}^{A, c}\right| \leq \overline{T_{m n}^{A, c}}$, for all time $t$, for all lines $m n$

(internal lines and tie - lines) of zone A, and for all zones

where $N_{m t}^{A, c}$ and $\theta_{m t}^{A, c}$ are the net injection and the phase angle of bus $m$ of zone A at time $t$ in case of contingency, respectively; $T_{m n, t}^{A, c}$ is the flow of line $m n$ (including internal lines and tie-lines) in zone A at time $t$ in the case of contingency. Observe that in this state transmission lines may carry two products, power and spinning reserve, at the same time with transmission emergency rating limits, $\overline{T_{m n}^{A, c}}$, being observed.

It should be noted that the constraints (29)-(35) are repeated in a similar way for the other areas. Therefore, the proposed model contains the objective function (1), all constraints (2)-(26), the collection of constraints (36)-(40) obtained from the linearization technique, described in the Appendix to linearize the reliability constraints, and the collection of constraints (29)-(35) for each control zone that are used for inter-zonal reserve trading modeling. The final model can be solved exhaustively using commercially available branch-and-cut software; for instance CPLEX [30].

\section{CASE STUDiES}

For the given data, the proposed model is programmed in linear form as addressed in [27]. We analyze two case studies including a six-bus and the IEEE-RTS test systems to illustrate the effectiveness of the proposed model.

\section{A. Six-Bus Test System}

This test system consists of three identical six-bus systems (namely areas A, B and C) which are interconnected together using three tie-lines. The configuration of the system chosen for the basic area $\mathrm{A}$ is depicted in Fig. 1. The most significant features of tie-lines are given in Table I.

Area $\mathrm{A}$ is considered as a basic area. The information of generating units and internal transmission lines of the basic area A are given in Tables II and III. Subsequently, the cost functions of area $\mathrm{B}$ and area $\mathrm{C}$ are defined respectively $120 \%$ and $80 \%$ of the cost function of the basic area. It is worth noting that the transmission emergency rating limits including tie-lines and internal lines of all control zones are assumed to be the same as the transmission normal capacity limits. Moreover, the generators are assumed to submit the spinning reserve offers at the price rates $q_{i t}$ equal to $50 \%$ of their $b$ coefficient. The failure rate of all generators and tie lines is supposed to be 0.002 . The 


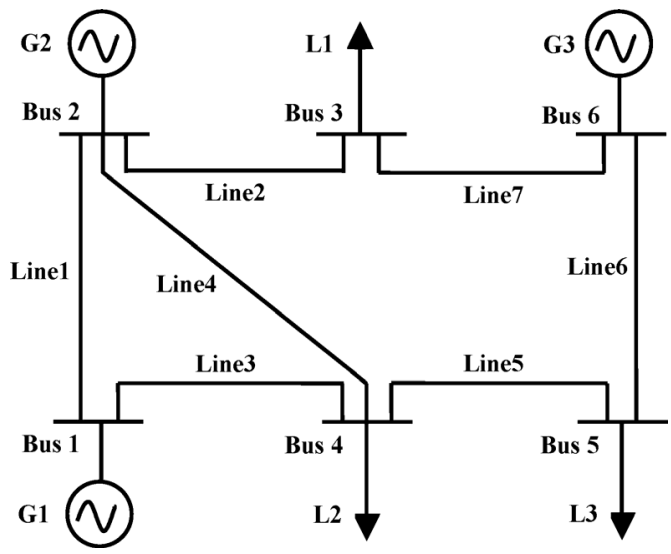

Fig. 1. One-line diagram of six-bus test system.

TABLE I

Tie-Lines Data-ThreE-Area Test System

\begin{tabular}{c|c|c|c|c}
\hline Tie-Line No. & From & To & $\mathrm{x}[\mathrm{pu}]$ & $T_{m n}^{A, \max }[M W]$ \\
\hline 1 & $A-4$ & $B-2$ & 0.154 & 200 \\
\hline 2 & $A-5$ & $C-1$ & 0.154 & 200 \\
\hline 3 & $B-3$ & $C-1$ & 0.154 & 200 \\
\hline
\end{tabular}

TABLE II

Generating Units Data-Basic AREa A

\begin{tabular}{|c|c|c|c|}
\hline & \multicolumn{3}{|c|}{ Generator } \\
\hline & 1 & 2 & 3 \\
\hline$P_{i}^{\min }[\mathrm{MW}]$ & 90 & 10 & 10 \\
\hline$P_{i}^{\max }[\mathrm{MW}]$ & 220 & 100 & 100 \\
\hline$a_{i}[\$]$ & 440 & 324 & 342 \\
\hline$b_{i}[\$ / \mathrm{MWh}]$ & 33.6 & 101.2 & 43.8 \\
\hline$c_{i}\left[\$ / M W^{2} h\right]$ & 0.001 & 0.0025 & 0.0125 \\
\hline$S_{i t}^{U}[\mathrm{MBtu}]$ & 124.7 & 250 & 0 \\
\hline$R_{i}^{\max }[\mathrm{MW}]$ & 100 & 100 & 100 \\
\hline
\end{tabular}

TABLE III

Transmission Lines Data—SiX-Bus Test System

\begin{tabular}{c|c|c|c|c}
\hline Line No. & From & To & $\mathrm{x}[\mathrm{pu}]$ & $L_{\operatorname{mn}}^{A, \max }[M W]$ \\
\hline 1 & 1 & 2 & 0.170 & 200 \\
\hline 2 & 2 & 3 & 0.037 & 100 \\
\hline 3 & 1 & 4 & 0.258 & 150 \\
\hline 4 & 2 & 4 & 0.197 & 150 \\
\hline 5 & 4 & 5 & 0.037 & 100 \\
\hline 6 & 5 & 6 & 0.140 & 100 \\
\hline 7 & 3 & 6 & 0.018 & 100 \\
\hline
\end{tabular}

generators' technical constraints (14) are assumed inactive here. The system is tested for a five-hour period. The load data for all control zones is assumed to be the same as shown in Table IV.

Inasmuch as ELNS accounts for both the outage probability and the corresponding average lost load, such a criterion is more appropriate than LOLP in power system operation. Hence, only the ELNS metric is used to set the required level of spinning reserve in this study. The ELNS bound is set to $0.2 \mathrm{MW}$ for all
TABLE IV

LOAD DATA OF EACH CONTROL ZONE

\begin{tabular}{cc|c|c|c|c}
\hline \multicolumn{6}{c|}{ Time $t[\mathrm{hr}]$} \\
\hline & $\begin{array}{c}1 \\
{[\mathrm{MW}]}\end{array}$ & $\begin{array}{c}2 \\
{[\mathrm{MW}]}\end{array}$ & $\begin{array}{c}3 \\
{[\mathrm{MW}]}\end{array}$ & $\begin{array}{c}4 \\
{[\mathrm{MW}]}\end{array}$ & $\begin{array}{c}5 \\
{[\mathrm{MW}]}\end{array}$ \\
\hline$L_{1 t}$ & 32.2 & 34.8 & 38 & 36.4 & 31.8 \\
\hline$L_{2 t}$ & 64.4 & 69.6 & 76 & 72.8 & 63.6 \\
\hline$L_{3 t}$ & 64.4 & 69.6 & 76 & 72.8 & 63.6 \\
\hline
\end{tabular}

TABLE V

MARKET-CLEARING RESULTS

\begin{tabular}{ccccccc}
\hline & & \multicolumn{6}{c}{ Time $t(\mathrm{~h})$} \\
\cline { 3 - 7 } Zones & & 1 & 2 & 3 & 4 & 5 \\
\hline \multirow{3}{*}{$\mathrm{A}$} & $P_{1 t}$ & 159.12 & 146.70 & 125.37 & 139.40 & 161.47 \\
& $R_{1 t}$ & 2.27 & 27.72 & 71.04 & 43.56 & 0 \\
\cline { 2 - 7 } & $R_{t}^{A}$ & 60.88 & 73.32 & 94.7 & 80.64 & 58.53 \\
\hline \multirow{3}{*}{$\mathrm{B}$} & $P_{1 t}$ & 102.57 & 128.57 & 154.68 & 144.06 & 96.47 \\
& $R_{1 t}$ & 58.61 & 45.6 & 23.66 & 37.08 & 58.53 \\
\cline { 2 - 7 } & $R_{t}^{B}$ & 60.88 & 73.32 & 76.93 & 79.01 & 58.53 \\
\hline \multirow{3}{*}{$\mathrm{C}$} & $P_{1 t}$ & 159.43 & 172.4 & 194.25 & 180.9 & 159.53 \\
& $P_{3 t}$ & 61.89 & 74.32 & 95.7 & 81.64 & 59.53 \\
\cline { 2 - 7 } & $R_{t}^{C}$ & 60.88 & 73.32 & 94.7 & 80.64 & 58.53 \\
\hline
\end{tabular}

TABLE VI

COMPARISON OF MARKET-CLEARING RESULTS-INTERCONNECTED VERSUS ISOLATED POWER SYSTEM

\begin{tabular}{|c|c|c|c|}
\hline Zones & & Interconnected & Isolated \\
\hline & $\tilde{R}^{A}$ & $16.7 \%$ & $41.5 \%$ \\
\hline \multirow[t]{2}{*}{ A } & $\tilde{P}^{A}$ & $84.5 \%$ & $100 \%$ \\
\hline & $\tilde{R}^{B}$ & $25.8 \%$ & $41.5 \%$ \\
\hline \multirow[t]{2}{*}{ B } & $\tilde{P}^{B}$ & $72.3 \%$ & $100 \%$ \\
\hline & $\tilde{R}^{C}$ & $0 \%$ & $41.5 \%$ \\
\hline $\mathrm{C}$ & $\tilde{P}^{C}$ & $143.1 \%$ & $100 \%$ \\
\hline \multicolumn{2}{|c|}{ System Total Cost [\$] } & 102153.908 & 134427.238 \\
\hline \multicolumn{2}{|c|}{ Average System LOLP [f/hr] } & 0.00266 & 0.00386 \\
\hline
\end{tabular}

control zones at each hour. Bus 1 in zone A is considered as the slack bus.

Table $\mathrm{V}$ outlines the market scheduling results including the detailed generation and spinning reserve for each control zone during the five-hour scheduling horizon. In order to save space, only the generators having been committed during the scheduling horizon are appearing in Table V. Table VI compares the market-clearing results determined in a multi-control zone power system with the results of the case when all areas are completely isolated from each other (the capacity of tie lines between areas are assumed to be $0 \mathrm{MW}$ ). It should be mentioned that $\tilde{P}^{A}$ and $\tilde{R}^{A}$ are the summation of power and spinning reserve provided in area A during the scheduling horizon, respectively. They are expressed in percentage of the load of their own zone (190 MW).

It can be realized from Table $\mathrm{V}$ and the first column of Table VI that the control zone $\mathrm{C}$, being incrementally the 
cheapest one, keeps a greater share of power production (around 143.1\% of its load) during the five-hour period without spinning reserve sharing in these periods; control zones A and B serve the remaining parts of system load, namely about $84.5 \%$ and $72.3 \%$ of their loads, respectively. A first conclusion would be that the power is flowing from inexpensive area to the costly one. In addition, the limited contribution of control zone B in power production on the one hand, and the capacity limitation of the other areas as well as the areas' reliability constraints on the other hand, enforce the control zone B to provide globally more spinning reserve than the other zones, as shown in the fifth and third rows of Tables V and VI, respectively. We note that the total required spinning reserve, $R_{t}^{A}$, is the summation of spinning reserve provided by its own area and the spinning reserve which the other areas can assist [see (12)]. Not surprisingly, the total required spinning reserve in each area is well correlated with the level of load demand of this area during the operational periods.

Table VI effectively illustrates that the inter-zonal trading in MAPS improves both the market efficiency and the system reliability by sharing the resources (power and reserve services) across zonal boundaries; in the isolated system, each area maintains about $41.5 \%$ of its load as spinning reserve and totally $124.5 \%$ (normalized based on the load of one control zone) for the whole system. In the interconnected one, all areas totally keep about $42.5 \%(16.7 \%+25.8 \%+0 \%)$ spinning reserve for the whole system. Hence, as expected, the total cost of operation in the interconnected power system is approximately $24 \%$ less than the operation cost of the isolated power system in this case study. In addition, comparing the average system LOLP of these two cases, we observe that this index in the multi-control zone system is lower than in the case of the isolated power system. So, one can say with certainty that the interconnected power system is more reliable than the isolated one.

In order to demonstrate the benefits of reserve sharing and not the energy arbitrage in the interconnected power system, a test case with similar spinning reserve offered cost but different generators operation cost as in the base case is considered. Herein, we scale up and down proportionally the coefficients of operation cost of the generators in zone $\mathrm{C}$ and $\mathrm{B}$, respectively, to have finally all generators with the same marginal cost as for the basic zone A. Fig. 2 demonstrates how the amount of generated power and spinning reserve of different areas vary under different scaling factors. It can be clearly seen from this figure that in the base case (scaling factor $20 \%$ ), area $\mathrm{C}$ has a big share in power production and zero share in spinning reserve provision. However, as the marginal cost of all areas come closer to each other, area $\mathrm{C}$, having the cheapest spinning reserve offer, participates more in spinning reserve provision. In other words, once the marginal cost of all areas are the same (scaling factor $0 \%$ ), each area is rather able to supply its own load demand by its own generators, and the required spinning reserve is provided more from the control zone with relatively low spinning reserve price (e.g., keep tracking of power and spinning reserve provision of zone $\mathrm{C}$ under various scaling factors).

Fig. 3 depicts how the provided spinning reserves within a given zone will be deployed and flow across the tie-lines to support the spinning reserve requirement of the other zones

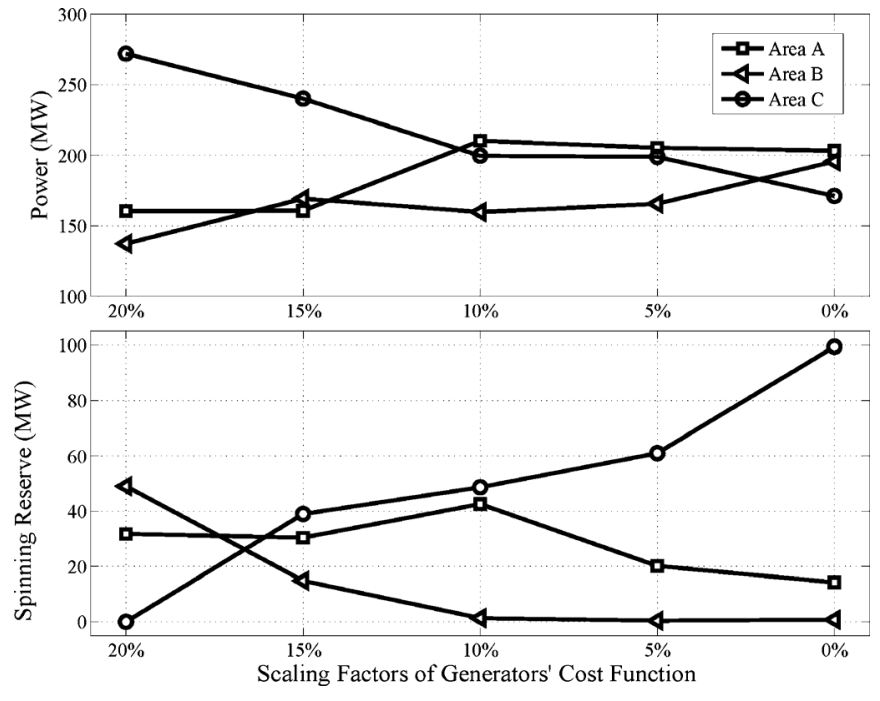

Fig. 2. Comparison of reserve sharing versus power sharing across the borders.

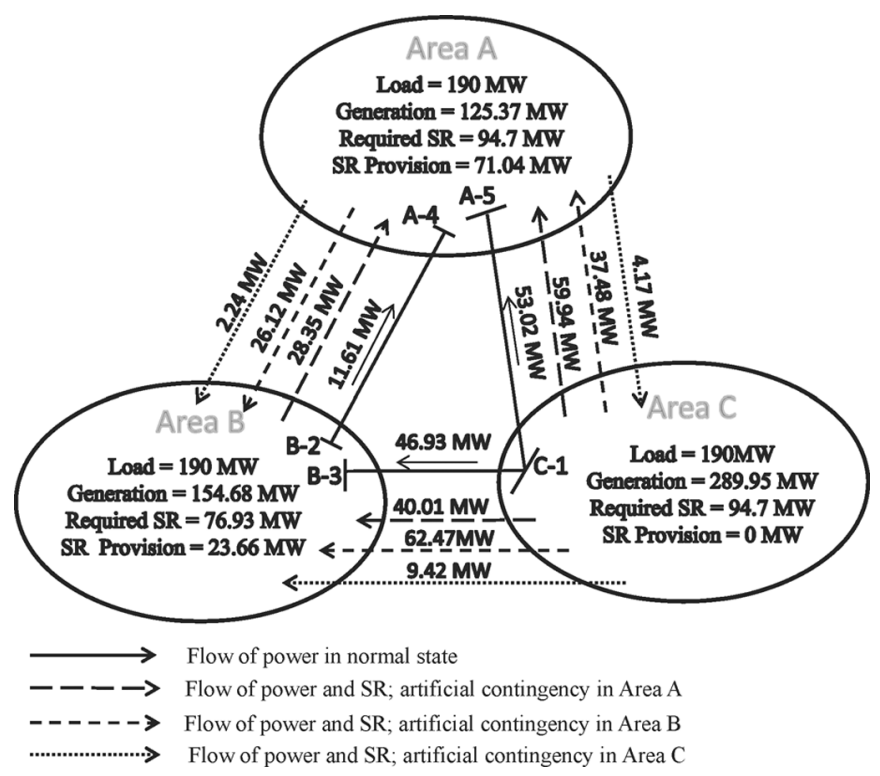

Fig. 3. Tie-line flows between areas in normal and contingency states.

determined by probabilistic criteria. As already mentioned in Section II-B, the tie-lines flows in different cases are obtained while contingency load flow constraints (29)-(32) are met. Note that each case corresponds to the occurrence of an artificial contingency in a given area.

Next, referring to Fig. 3, we analyze in detail the contingency load flow equations for checking tie-lines' flows. Consider the case of area $\mathrm{A}$ at the peak load time, for instance. We observe that area A generates $125.37 \mathrm{MW}$ by its generators, imports 53.02 MW and 11.61 MW from area $\mathrm{C}$ and area B, respectively, in order to satisfy the load of its area $190 \mathrm{MW}$. In addition, the probabilistic criteria (here, ELNS) impose that 94.7 MW spinning reserve has to be provided in order to respond to the expected contingency in this area; $71.04 \mathrm{MW}$ of this required spinning reserve is provided by unit $G_{1}$ of this area and the remaining $23.66 \mathrm{MW}$ is provided by $G_{1}$ of area B. Note that area $\mathrm{C}$ does not provide spinning reserve at all. Actually, the tie-lines flows, 28.35 MW from area B to A, 59.94 MW from area C to A 


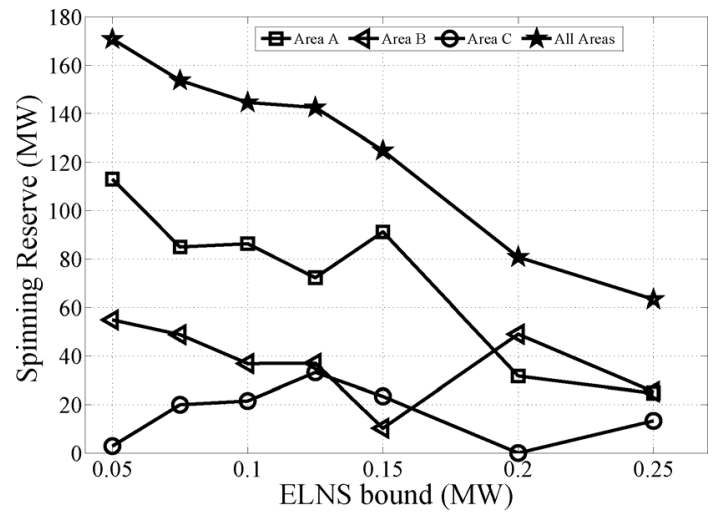

Fig. 4. Variation of spinning reserve versus ELNS bound.

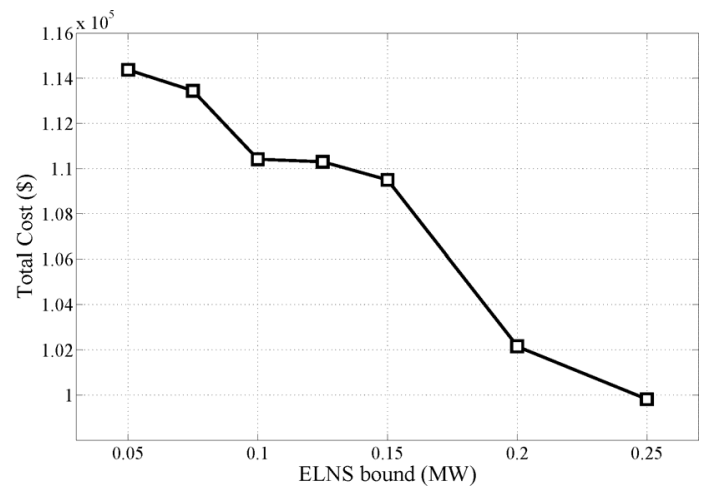

Fig. 5. Variation of total cost versus ELNS bound.

and 40.01 MW from area $\mathrm{C}$ to $\mathrm{B}$, resulting from the contingency load flow of area A indicate how the 23.66 MW spinning reserve will flow across the tie-lines to assist area A when it is needed. In this case, area B activates $23.66 \mathrm{MW}$ of its spinning reserve; it exports directly $16.74 \mathrm{MW}[28.35 \mathrm{MW}-(11.61) \mathrm{MW}]$ of this spinning reserve to area A via tie-line 1 and it exports indirectly 6.92 MW (inducing 6.92 MW counterflow) to area A via area C. It can be addressed in such a way that the area $\mathrm{C}$ decreases its normal export to area B of 6.92 MW (from 46.93 MW to 40.01 MW) whereas it increases of $6.92 \mathrm{MW}$ its export to A (from 53.02 MW to 59.94 MW). It is reminded that tie-lines flows in the contingency states include both flows of power and spinning reserve at the same time.

One of the most important factors influencing the required quantity of spinning reserve and the system total cost is the ELNS metric. It is observed from Fig. 4 that the spinning reserve, as expected, increases as we tighten the ELNS metric bound; as consequence, the total operation cost increases as the level of spinning reserve increases; i.e., ELNS metric bound decreases. This circumstance is demonstrated in Fig. 5.

\section{B. IEEE-RTS Simulation}

The model is also tested over a 24-h scheduling horizon on the IEEE-RTS [31]. The data for the generators and the loads have been extracted from [32]. All generators offer the possible amount of spinning reserve at a price rate equal to the coefficient $b$ of their cost function. The hourly demand data correspond to a Thursday of a winter (week 45) with the annual peak load
TABLE VII

COMPARISON OF MARKET-CLEARING RESULTS OF IEEE-RTS-INTERCONNECTED VERSUS ISOLATED POWER SYSTEM

\begin{tabular}{|c|c|c|c|}
\hline Zones & & Interconnected & Isolated \\
\hline & $\tilde{R}^{A}$ & $1.7 \%$ & $6 \%$ \\
\hline \multirow[t]{2}{*}{ A } & $\tilde{P}^{A}$ & $103.3 \%$ & $100 \%$ \\
\hline & $\tilde{R}^{B}$ & $3.4 \%$ & $6 \%$ \\
\hline \multirow[t]{2}{*}{ B } & $\tilde{P}^{B}$ & $85 \%$ & $100 \%$ \\
\hline & $\tilde{R}^{C}$ & $1.8 \%$ & $6 \%$ \\
\hline $\mathrm{C}$ & $\tilde{P}^{C}$ & $111.7 \%$ & $100 \%$ \\
\hline \multicolumn{2}{|c|}{ System Total Cost [\$] } & 1395319.67 & 1511339.603 \\
\hline \multicolumn{2}{|c|}{ Average System LOLP [f/hr] } & 0.0023 & 0.00312 \\
\hline
\end{tabular}

of $2850 \mathrm{MW}$ ( $8550 \mathrm{MW}$ for the whole multi-control zone test system). The ELNS metric bound is set to $0.5 \mathrm{MW}$ for all control zones. In order to impose inter-zonal trading among the areas, the cost functions of zones B and C are defined, respectively, $20 \%$ greater and $20 \%$ lower than the cost function of the basic zone A. Moreover, the rating of aggregate tie-lines between all control zones are set to $350 \mathrm{MW}$.

Table VII presents the comparison of market-clearing results in an interconnected power system versus an isolated power system. It is observed from this table that zone B, being incrementally the expensive one, keeps a less share of power production ( $85 \%$ of its own load) during the $24 \mathrm{~h}$ and, subsequently, control zones A and C serve some parts of the load of this area. On the other hand, zone B provides the most parts of spinning reserve requirement for the whole system. This is due to the fact that zone B keeps the lowest share of power production; it has, therefore, more available resources for reserve production than the others. In contrast, although control zone $\mathrm{C}$ has the cheapest spinning reserve offer cost, it only provides a small part of system spinning reserve requirement (1.8\% of its load) as it participates more in power production $(111.7 \%$ of its own load).

It is also interesting to observe the effect of inter-zonal spinning reserve trading which has been highlighted once the provided spinning reserve between the interconnected and isolated cases are compared. In the isolated power system, every zone has to keep on average $6 \%$ of its load as spinning reserve to satisfy the reliability criteria (totally $18 \%$ for the whole system), but in the interconnected one, this amount decreases to $6.9 \%$ for the whole system. Therefore, as expected, the global operation cost in the case of interconnected system is much lower than the operation cost of the isolated system. In addition, comparing the LOLP index of these two cases confirms that the interconnected system is more reliable than the isolated one.

It is also helpful to investigate the computation time of the proposed algorithm. The computation time is less than one hour on a $2.67-\mathrm{GHz}$ processor (personal computer) with 4GB-RAM, which is almost tolerable in day-ahead market environment. In spite of the limited size of IEEE-RTS, the dimension of the corresponding problem is important. We note, however, that some modifications such as performance of computing machinery, 
optimization codes, decomposition algorithms can be made to speed up the computational time.

\section{CONCLUSION}

We propose in this paper an efficient algorithm for probabilistic spinning reserve assessment in multi-control zone power system. Different control zones can assist each other when a contingency occurs in an area, where it cannot prevent its load from disconnection. Therefore, as demonstrated in the numerical results, this assistance may decrease the required spinning reserve and consequently increase the financial benefit in the interconnected power system. The non-coincidental nature of spinning reserve requirement is effectively handled by introducing the notation of artificial contingency load flow for each zone. The most important advantage of this proposed model is that the reliability metrics can be easily incorporated into SCUC formulation and also conveniently programmed with powerful MILP tools such as CPLEX. Finally, we illustrated the performance of the proposed model using a small-scale example and a realistic case study; the obtained results conclude that:

1) The inter-zonal trading in a multi-control zone power system improves the system efficiency from both the economic and security point of view by sharing the resources (energy and spinning reserves ) across the boundaries.

2) The simulation results for both case studies, small-scale test system and IEEE-RTS, are consistent.

The present work is under way to be investigated in a decentralized MAPS.

\section{APPENDIX \\ LINEARIZATION OF THE LOLP AND ELNS METRICS}

The linearization technique presented in [28] and [33] is used in our proposed model 1) to transform a product of binary variables in (17) and (18) into a set of additional inequalities with a new continuous variable, and 2) to express the bilinear product of a $0-1$ variable and a continuous variable in (18) as a set of additional linear constraints.

Let $x$ be the product of $n$ binary variables $u_{1}, \ldots, u_{n}$. Assuming that $x$ is a continuous variable, this product $\left(x=\prod_{i=1}^{n} u_{i}\right)$ is equivalent to the $n+2$ following linear inequalities:

$$
\begin{aligned}
& x \geq 0, \\
& x \leq u_{i}, i=1, \ldots, n \\
& x \geq \sum_{i}^{n} u_{i}-n+1 .
\end{aligned}
$$

Generally, the basic idea for modeling the bilinear product of a bounded continuous variable $y \in\left[y^{\min }, y^{\max }\right]$ and $0-1$ variable $x$ is to introduce a new continuous variable $h$ such that $h=x . y$. This product is equivalent to the additional linear constraints as follows:

$$
\begin{aligned}
x y^{\min } & \leq h \leq x y^{\max }, \\
y-y^{\max }(1-x) & \leq h \leq y-y^{\min }(1-x) .
\end{aligned}
$$

Note that if $x=0$, then the first constraint implies that $h=0$, while the second constraint is relaxed. Otherwise, when $x=1$, the second constraint implies that $h=y$, while the first constraint is inactive.

\section{REFERENCES}

[1] J. Wood and B. F. Wollenberg, Power Generation, Operation and Control. New York, NY, USA: Wiley, 1996.

[2] A. A. Khatir and R. Cherkaoui, Fundamental Issues on Frequency Control Reserves Provision, EPFL Lausanne, Power System Group, Lausanne, Switzerland, 2010, Tech. rep.

[3] R. Allan and R. Billinton, "Probabilistic assessment of power systems," Proc. IEEE, vol. 88, no. 2, pp. 140-162, Feb. 2000.

[4] R. Billinton and R. N. Allan, Reliability Evaluation of Power Systems, 2nd ed. New York, NY, USA: Plenum, 1996.

[5] L. T. Anstine, R. E. Burke, J. E. Casey, R. Holgate, R. S. John, and H. G. Stewart, "Application of probability methods to the determination of spinning reserve requirements for the Pennsylvania-New Jersey-Maryland interconnection," IEEE Trans. Power App. Syst., vol. PAS-82, no 68 , pp. $726-735$, Oct. 1963.

[6] R. Doherty and M. O'Malley, "A new approach to quantify reserve demand in systems with significant installed wind capacity," IEEE Trans. Power Syst., vol. 20, no. 2, pp. 587-595, May 2005.

[7] C. Maurer, S. Krahl, and H. Weber, "Dimensioning of secondary and tertiary control reserve by probabilistic methods," Eur. Trans. Elect. Power, vol. 19, pp. 544-552, 2009.

[8] J. D. Guy, "Security constrained unit commitment," IEEE Trans. Power App. Syst., vol. PAS-90, no. 3, pp. 1385-1390, May 1971.

[9] R. Allan and R. Billinton, "Power system reliability and its assessment. iii. Distribution systems and economic considerations," Power Eng. J., vol. 7, no. 4, pp. 185-192, Aug. 1993.

[10] F. N. Lee, Q. Chen, and A. Breipohl, "Unit commitment risk with sequential rescheduling," IEEE Trans. Power Syst., vol. 6, no. 3, pp. 1017-1023, Aug. 1991.

[11] H. B. Gooi, D. P. Mendes, K. R. W. Bell, and D. S. Kirschen, "Optimal scheduling of spinning reserve," IEEE Trans. Power Syst., vol. 14, no. 4, pp. 1485-1492, Nov. 1999.

[12] A. Ahmadi-Khatir, M. Fotuhi-Firuzabad, and L. Goel, "Customer choice of reliability in spinning reserve procurement and cost allocation using well-being analysis," Elect. Power Syst. Res., pp. 1431-1440, May 2009.

[13] M. A. Ortega-Vazquez and D. S. Kirschen, "Optimizing the spinning reserve requirements using a cost/benefit analysis," IEEE Trans. Power Syst., vol. 22, no. 1, pp. 24-33, Feb. 2007.

[14] D. Chattopadhyay and R. Baldick, "Unit commitment with probabilistic reserve," in Proc. IEEE Power Engineering Society Winter Meeting, 2002, vol. 1, pp. 280-285.

[15] M. A. Ortega-Vazquez, D. S. Kirschen, and D. Pudjianto, "Optimising the scheduling of spinning reserve considering the cost of interruptions," Proc. Inst. Elect. Eng., Gen., Transm., Distrib., vol. 153, no. 5, pp. 570-575, 2006.

[16] F. Bouffard and F. D. Galiana, "An electricity market with a probabilistic spinning reserve criterion," IEEE Trans. Power Syst., vol. 19, no. 1, pp. 300-307, Feb. 2004.

[17] F. Aminifar, M. Fotuhi-Firuzabad, and M. Shahidehpour, "Unit commitment with probabilistic spinning reserve and interruptible load considerations," IEEE Trans. Power Syst., vol. 24, no. 1, pp. 388-397, Feb. 2009.

[18] F. Bouffard, F. D. Galiana, and A. J. Conejo, "Market-clearing with stochastic security-part i: Formulation," IEEE Trans. Power Syst., vol. 20, no. 4, pp. 1818-1826, Nov. 2005.

[19] A. Lamadrid, S. Maneevitjit, T. D. Mount, C. Murillo-Saanchez, R. J. Thomas, and D. Zimmerman R., A Superopf Framework, Cornell University, Ithaca, NY, USA, 2008, Tech. rep.

[20] J. M. Morales, A. J. Conejo, and J. Perez-Ruiz, "Economic valuation of reserves in power systems with high penetration of wind power," IEEE Trans. Power Syst., vol. 24, no. 2, pp. 900-910, May 2009.

[21] A. A. Khatir and R. Cherkaoui, "Preventive and corrective security market model," in Proc. 17th Power Systems Computation Conf. Stockholm, Sweden, 2011.

[22] P. A. Ruiz, C. R. Philbrick, E. Zak, K. W. Cheung, and P. W. Sauer "Uncertainty management in the unit commitment problem," IEEE Trans. Power Syst., vol. 24, no. 2, pp. 642-651, May 2009. 
[23] J. Adapa, R. Lee, and F. N. Huang, "Multi-area unit commitment via sequential method dc power flow network model," IEEE Trans. Power Syst., vol. 9, no. 1, pp. 279-287, Feb. 1994.

[24] C.-L. Tseng, X. Guan, and A. J. Svoboda, "Multi-area unit commitment for large-scale power systems," Proc. Inst. Elect. Eng., Gen., Transm., Distrib., vol. 145, no. 4, pp. 415-421, Jul. 1998.

[25] C. Yingvivatanapong, L. Wei-Jen, and E. Liu, "Multi-area power generation dispatch in competitive markets," IEEE Trans. Power Syst., vol. 23, no. 1, pp. 196-203, Feb. 2008.

[26] E. G. Read, Co-optimization of Energy and Ancillary Services, Handbook of Power Systems Part I. New York, NY, USA: Springer, 2010, pp. 307-327.

[27] M. Carrion and J. M. Arroyo, "A computationally efficient mixed-integer linear formulation for the thermal unit commitment problem," IEEE Trans. Power Syst., vol. 21, no. 3, pp. 1371-1378, Aug. 2006.

[28] H. P. Williams, Model Building in Mathematical Programming. New York, NY, USA: Wiley, 1999.

[29] H. P. Williams, Logic and Integer Programming. New York, NY, USA: Springer, 2009.

[30] R. E. Rosenthal, Gams-A User's Guide, GAMS Development Corp., Washington, DC, 2010, Technical report.

[31] Reliaility Test System Task Force, "The IEEE reliability test system 1996," IEEE Trans. Power Syst., vol. 14, no. 3, pp. 1110-1120, Aug. 1999.

[32] M. Ortega-Vazquez, "Optimizing the spinning reserve requirements," Ph.D. dissertation, Univ. Manchester, Manchester, U.K., 2006.

[33] C. A. Floudas, Nonlinear and Mixed-Integer Optimization: Fundamentals and Applications. New York, NY, USA: Oxford Univ. Press, 1995.

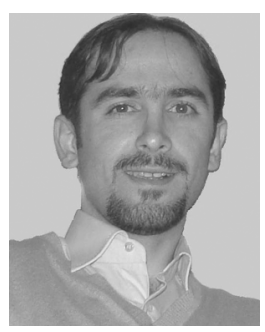

Ali Ahmadi-Khatir (S'09) received the M.Sc. degree from Sharif University of technology, Tehran, Iran, in 2005 . He is currently pursuing the Ph.D. degree at the EPFL, Lausanne, Switzerland.

His research interests include electricity markets and power system optimization.

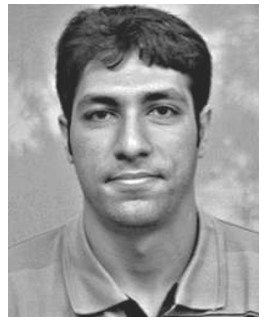

Mokhtar Bozorg (S'12) received the M.Sc. degree from Sharif University of technology, Tehran, Iran, in 2011 . He is currently pursuing the Ph.D. degree at the EPFL, Lausanne, Switzerland.

His research interests include electricity markets and power system optimization.

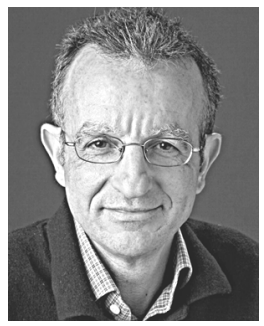

Rachid Cherkaoui (M'05-SM'07) received the M.S. and Ph.D. degrees from EPFL, Lausanne, Switzerland, in 1983 and 1992, respectively.

$\mathrm{He}$ is currently the head of power system group at EPFL. His research interests include electricity market regulation, distributed generation and storage and power system vulnerability mitigation. $\mathrm{He}$ is the author of more than 80 scientific publications. 